\title{
Analysis of Existing and Proposed 3-Bit and Multi-Bit Multiplier Algorithms for FIR Filters and Adaptive Channel Equalizers on FPGA
}

\author{
Aneela Pathan ${ }^{1, *}$, Tayab Din Memon ${ }^{1}$, Fareesa Khan Sohu ${ }^{2}$, Muhammad Awais Rajput ${ }^{3}$ \\ ${ }^{1}$ Department of Electronic Engineering, MUET, Jamshoro, Pakistan. \\ ${ }^{2}$ Department of Electronic Engineering, QUEST, Nawabshah, Pakistan. \\ ${ }^{3}$ Department of Artificial Intelligence, QUEST, Nawabshah, Pakistan. \\ ${ }^{*}$ Corresponding author: pathan_aneela@quest.edu.pk
}

\section{Abstract}

Different multiplication algorithms have different performance characteristics. Some are good at speed while others consume less area when implemented on hardware, like Field Programmable Gate Array (FPGA)-the advanced implementation technology for DSP systems. The eminent parallel and sequential multiplication algorithms include Shift and Add, Wallace Tree, Booth, and Array. The multiplier optimization attempts have also been reported in adders used for partial product addition. In this paper, analogous to conventional multipliers, two new multiplication algorithms implemented on FPGA are shown and compared with conventional algorithms as stand-alone and by using them in the implementation of FIR filters and adaptive channel equalizer using the LMS algorithm. The work is carried out on Spartan-6 FPG that may be extended for any type of FPGA. Results are compared in terms of resource utilization, power consumption, and maximum achieved frequency. The results show that for a small length of coefficients like 3-bit, the proposed algorithms work very well in terms of achieved frequency, consumed power, and even resource utilization. Whilst for the length greater than 3-bit, the Pipelined multiplier is much better in frequency than the proposed and conventional ones, and the Booth multiplier consumes fewer resources in terms of lookup tables.

Keywords-Adaptive channel equalize, FPGA, FIR filter, LMS, multiplier

\section{Introduction}

$\mathrm{V}$ LSI is good for the fast implementation of complex DSP functions [1]. On the other hand, it is a challenging task to implement complex DSP systems with small areas, optimal performance, and minimal power usage, especially, in battery dependent portable devices, like mobile phones [2]. Researchers around the world have accepted that challenge and are working towards solutions especially to fulfill difficult computing requirements[3]. In simplifying the multiplication operation, various traditional and non-traditional multiplier encoding techniques are reported including Booth's Algorithm[4], Wallace Tree Multipliers [5], DADA Multipliers[6], and Vedic Multipliers[7]. Multiplier optimization attempts have also been reported in adders. Some well-known methods are Carry-Save

ISSN: 2523-0379 (Online), ISSN: 1605-8607 (Print)

DOI: https://doi.org/10.52584/QRJ.1901.12

This is an open access article published by Quaid-e-Awam University of Engineering Science \& Technology, Nawabshah, Pakistan under CC BY 4.0 International License.
Adder [8], Carry Look-Ahead, and Carry-skip Adders.

Another method to reduce the size of hardware is distributed arithmetic that has been adopted in FPGA-based implementation for many years (Here, the stages of performing the multiplication are carried out using look-up tables) [9]. In DSP algorithm optimization, a major bottleneck is the multiplier complexity that is evident for example in FIR, IIR, FFT, etc. Despite current optimization proposals, multiplier implementations still take more area and consume more power and observe delay[10-12].

The authors aim to continue the work in the domain of multiplier optimization and to validate the performance of existing state-of-the-art architectures. Besides, the implementation of two new proposed algorithms is also carried out and compared with the conventional architectures. It is shown that amongst the existing and proposed designs, the Pipelined multiplier shows superior performance in frequency and the Booth multiplier consumes fewer resources; hence, 
showing the area-speed trade-off.

The paper further proceeds as follows. In Section 2 , the architecture of state-of-the-art and proposed multipliers is discussed in a 3-bit configuration that may be further extended to multi-bits for FPGAbased implementation and comparison. Section 3 gives the review of the FIR filter and channel equalizer. Section 4 discusses the implementation of FIR filter and channel equalizer. The power analysis of various adders and multipliers is carried out in section 5 , and section 6 concludes the paper.

\section{Conventional \& Proposed Multipliers}

The following sections describe the conventional multipliers and our proposed multiplier.

\subsection{Memory Based Multiplier}

The FPGA-based implementation of a memory-based multiplier is possible in two ways: i) using the RAM/ROM module, and ii) the look-up table based design [13-14]. The two common approaches in LUTbased memory design are: i) using Direct-LUT to compute the multiplication [15-21], and ii) to compute the inner product using Distributed Arithmetic (DA) [2226]. In Direct-LUT-based computation, all the possible product terms of the input multiplicand with the fixed coefficients are pre-computed and stored directly in the LUT, and thus, the multiplication is performed [27]. In DA-based computation, the N-point vector's inner product with the N-bit vector is pre-computed and stored in LUT [22]. LUT's size increases with the word length of the input if the product term is directly stored in LUT. Whereas, if the inner product is stored, the size increases with the length of the inner product $[28]$.

As the memory-based multiplier requires an adequate amount of memory of size $22 L$, where $L$ is the word length of the operands. Most of the work in the research leads to the design of a fixed-coefficient multiplier (leading to memory reduction from $22 \mathrm{~L}$ to $2 L$ ). One of the earlier techniques to implement fixed-coefficient multipliers using lookup table based memory of FPGA's was developed by Xilinx [29]. This relies on lookup tables rather than a network of adders to perform most of the multiplication. It is evident that there are sixteen possible results when a 4-bit number is multiplied by an 8-bit fixed number (because there are sixteen different four-bit numbers). Thus, a 4-bit variable times 8-bit constant multiplier can be implemented by a 16-entry lookup table. Each entry must be 12 bits (the width of the largest possible output). This

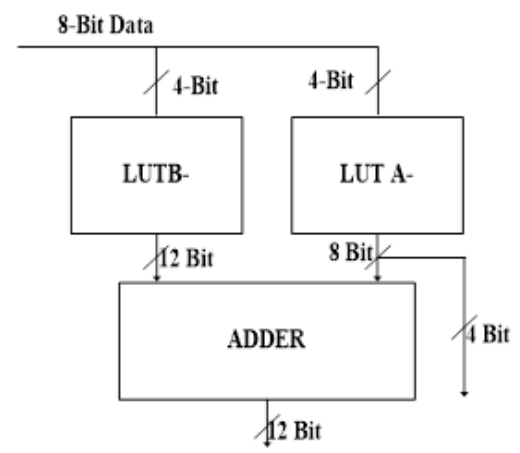

Fig. 1: Basic LUT based constant multiplier

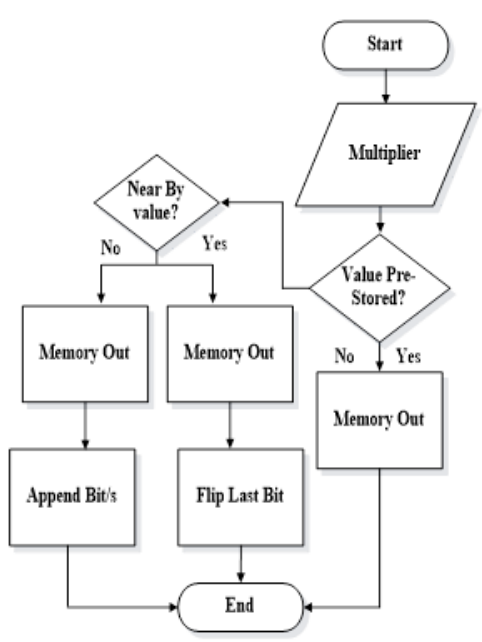

Fig. 2: Flow diagram of the proposed LUT-based multiplier

idea may easily be understood as follows. Let the 8bit constant multiplier is $25010\left(11111010_{2}\right)$, and 4-bit variable multiplicands are 0-15(0000-1111). The LUT entries are shown in Table 1. With the same approach, an $8 \times 8$ bit constant multiplier may be built using two of these $4 \times 8$ bit constant multipliers in the configuration shown in Figure 1. In [30], authors have proposed a new LUT-based multiplier, where three different $3 \times 3$ constant-coefficient unsigned integral multipliers were designed; two using FPGA's built-in primitives (block RAM and DSP48 based multiplier) and third with a proposed design using LUT-based implementation. The flow chart of the proposed multiplier and the FPGA-based implementation diagram is given in Figure 2 and Figure 3, respectively. The pseudo code of their proposed algorithm is given in Algorithm 1.

As the FPGA works on the modular approach, therefore, this 3-bit configuration was instantiated in the Xilinx ISE project accordingly to get the 8-bit implementation of the proposed algorithm. 


\begin{tabular}{|c|c|c|c|}
\hline *MP & ${ }^{*} \mathrm{MC}$ & ${ }^{*} \mathrm{PR}$ & 12-bit-value stored in LUT \\
\hline \multirow{17}{*}{250} & $0(0000)$ & 0 & LUT0 $=000000000000$ \\
\hline & $1(0001)$ & 250 & LUT1 $=000011111010$ \\
\hline & $2(0010)$ & 500 & LUT2 $=000111110100$ \\
\hline & $3(0011)$ & 750 & LUT3 $=001011101110$ \\
\hline & $4(0100)$ & 1000 & LUT4 $=001111101000$ \\
\hline & $5(0101)$ & 1250 & LUT $5=010011100010$ \\
\hline & $6(0110)$ & 1500 & LUT6 $=010111011100$ \\
\hline & $7(0111)$ & 1750 & LUT7 $=011011010110$ \\
\hline & $8(1000)$ & 2000 & LUT8 $=011111010000$ \\
\hline & $9(1001)$ & 2250 & LUT9 $=100011001010$ \\
\hline & $10(1010)$ & 2500 & LUT10 $=100111000100$ \\
\hline & $11(1011)$ & 2750 & LUT11 $=101010111110$ \\
\hline & $12(1100)$ & 3000 & LUT12=101110111000 \\
\hline & $13(1101)$ & 3250 & LUT13 $=110010110010$ \\
\hline & $14(1110)$ & 3500 & LUT14=1101101011100 \\
\hline & $15(1111)$ & 3750 & LUT15 = 111010100110 \\
\hline & \multicolumn{3}{|c|}{${ }^{*} \mathrm{MP}=$ multiplier ${ }^{*} \mathrm{MC}=$ multiplicand,${ }^{*} \mathrm{PR}=$ Product value } \\
\hline
\end{tabular}

TABLE 1: LUT values for a constant multiplier $\left(250_{10}\right)$

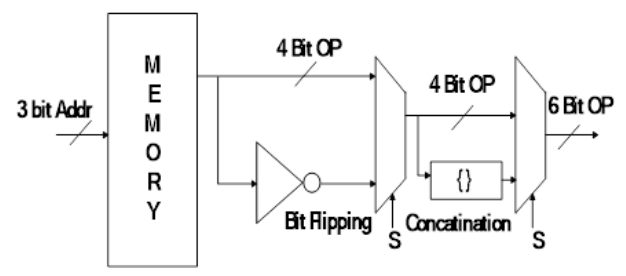

Fig. 3: FPGA based system design

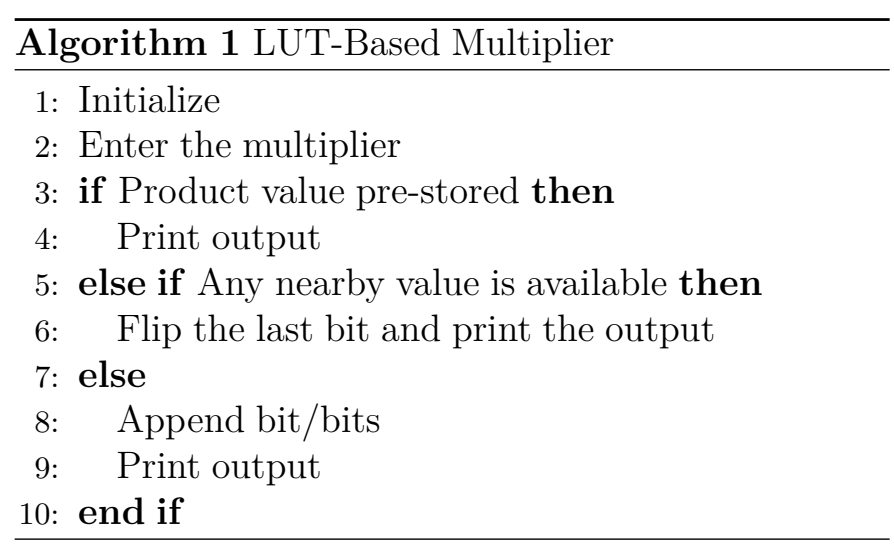

\subsection{Shift/Add Multiplier}

The traditional method of multiplication is the shift and add. In this method, depending on the value of the multiplier's LSB bit, a value of the multiplicand is added and accumulated [31]. As shown in Figure 5, at each clock cycle, the multiplier is shifted one bit to the right and its value is tested. If it is 0 , then only a shift operation is performed on the accumulator. If the value is 1 , then the multiplicand is added to the accumulator and is shifted by one bit to the right. After all the multiplier bits have been tested, the product is stored in the accumulator. The accumulator is $2 N(M+N)$ in size and initially $N$ LSBs contain the multiplier [32].

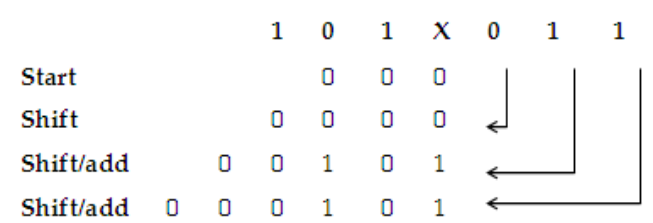

Fig. 4: General logic of shift/add multiplier

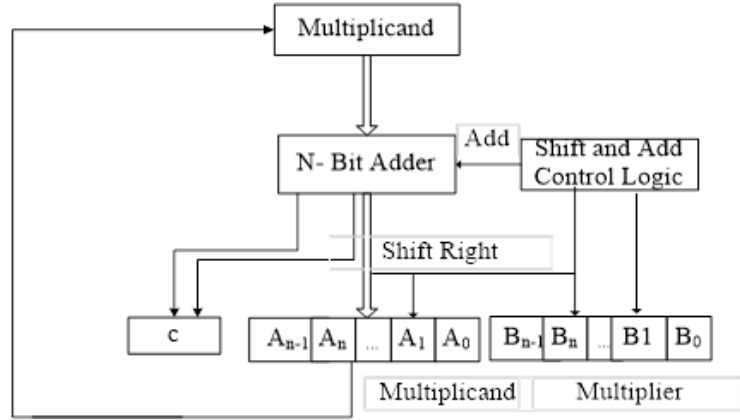

Fig. 5: Shift/Add multiplier block diagram

The delay is of $N$ cycles at maximum. The logic may be observed in Figure 4. The implementation of the algorithm consumes more resources, as it creates multiplier elements via shift and adds operations (Figure 4) [33]. Hence, this approach is specifically used when the number of DSP elements on an FPGA is exhausted (none are available) or when the throughput is not given much consideration [34].

In [35], authors have proposed a new way to perform shift and add multiplication and have implemented the design using FPGA (Figure 6). The algorithm of their proposed multiplier is given as follows.

- For the $3 \times 3$ multiplier, the operands take the values $0,1,2$, and 3 . 


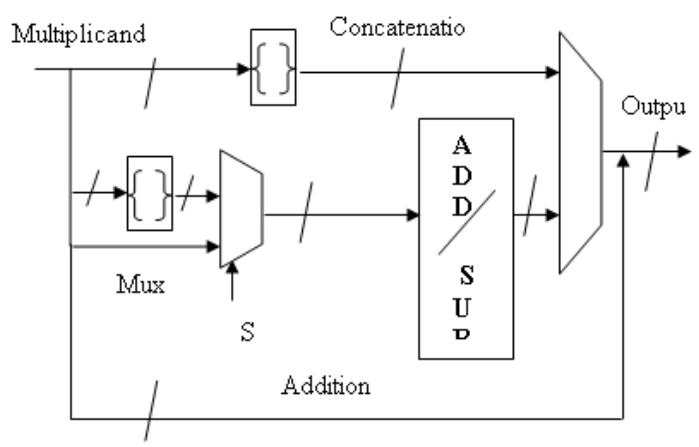

Fig. 6: FPGA based system design of proposed shift and add the multiplier

- If the multiplier is zero for any multiplicand, the product will be zero.

- If the multiplier is one for any multiplicand, the product will be same as the multiplicand.

- For the multiplier value 2 , just append a zero at the output of the multiplicand.

- For the multiplier value 3, append a zero to the multiplicand and add it to the actual multiplicand.

The pseudo code is given as follows.

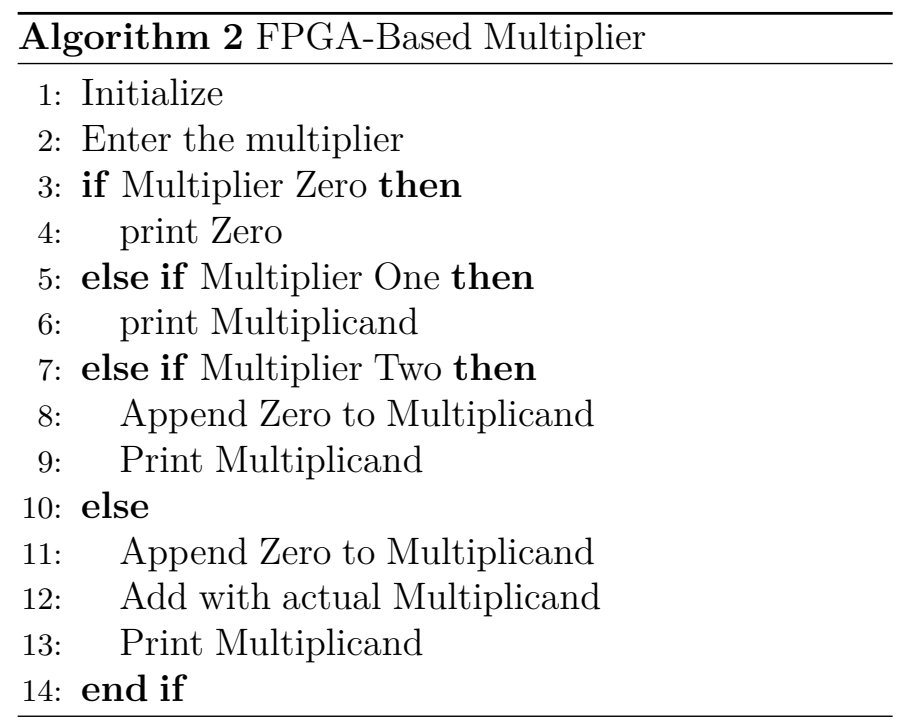

The 3-bit design of the proposed method was further extended to 8- bit for comparison with the state of art architectures. The FPGA-based implementation alone and for being used in FIR and channel equalizer of the proposed multipliers is given in the proceeding sections.

\section{FIR Filter \& the Channel Equalizer}

In digital signal processing, one of the well familiar filters is Finite Impulse Response (FIR) [36] whose response to any input is of finite duration or infinite time

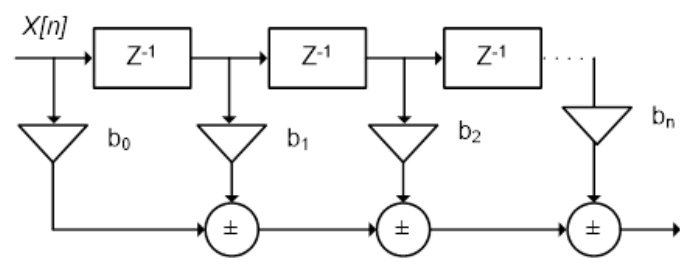

Fig. 7: A Direct Form Representation of Discrete-Time FIR Filter of Order N

duration [17]. Whereas, in contrast to this, another filter is Infinite Impulse Response filter (IIR) which has the feedback loop in the internal architecture. Unlike FIR, IIR continues to respond indefinitely (usually decaying). Four characterizations of FIR may be digital or analog, or continuous-time, and digital. The impulse response of an $\mathrm{N}^{\text {th }}$ order discrete-time FIR filter lasts exactly $N+1$ samples (from first nonzero element through last nonzero element) before it then settles to zero. Channel equalization is a common phenomenon of wireless communication. It is observed that in multipath propagation, when more than one copy of the transmitted signal is achieved at the receiver side, they result in inter-symbol interference (ISI) [18]. The channel can be modeled as a finite-impulse- response, linear filter and to get out of ISI, a counter FIR is used whose weights get updated adaptively [19]. This is known as adaptive channel equalization.

\section{FPGA Based Implementation \& Results}

The implementation consists of five phases. In the first phase, all the available adder circuits are designed and synthesized in their conventional architectures. In the second phase, proposed multipliers in a 3-bit configuration are designed and compared. After that, all the 8-bit conventional and proposed multipliers are implemented stand-alone and then used in the design of an 11-tap FIR filter, and in the final, all multiplier architectures are used for designing the LMS algorithm for adaptive channel equalizer. The simulations and synthesis are carried out on Xilinx Spartan-6 FPGA, and the factors for comparison are taken as the lookup tables (LUTs), DSP48 built-in core, Block Ram, delay observed, total logic levels, and the maximum achieved frequency.

\subsection{FPGA Based Implementation of Various Adder Circuits}

In multiplier optimization attempts are also reported in adders that are used for partial product additions. Some well-known methods implemented in this work 


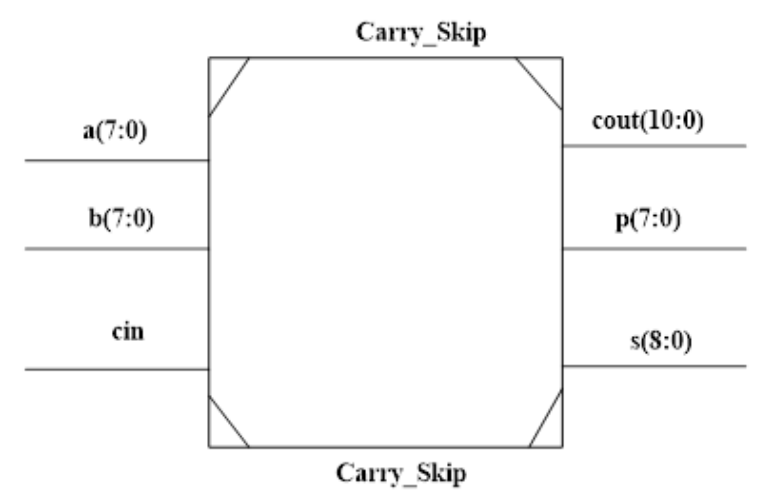

Fig. 8: RTL view of Carry-Skip Adder

\begin{tabular}{|l|l|l|l|l|}
\hline Factors & $\begin{array}{l}\text { Carry } \\
\text { Save } \\
\text { Adder }\end{array}$ & $\begin{array}{l}\text { Carry } \\
\text { Skip } \\
\text { Adder }\end{array}$ & $\begin{array}{l}\text { Carry } \\
\text { Look-Ahead } \\
\text { Adder }\end{array}$ & $\begin{array}{l}\text { Carry } \\
\text { Select } \\
\text { Adder }\end{array}$ \\
\hline LUTs & 35 & 24 & 15 & 18 \\
\hline Delay(ns) & 10.831 & 9.141 & 8.980 & 8.558 \\
\hline $\begin{array}{l}\text { Logic } \\
\text { Level }\end{array}$ & 8 & 5 & 5 & 5 \\
\hline $\begin{array}{l}\text { Frequency } \\
\text { (MHz) }\end{array}$ & 92.3 & 109.3 & 111.35 & 115.233 \\
\hline
\end{tabular}

TABLE 2: FPGA based implementation of various adder circuits

are carry-save adder, cary look-ahead adder, carry skip adder, and carry select adder. The length of the adders is set to 8-bits. Xilinx ISE 14.2 is used for the synthesis of the design. The RTL view of the carry skip adder is shown in Figure 7, and the FPGA-based implementation of various adder circuits is given in Table 2. The implementation results show that the carry look-ahead adder consumes a fewer number of lookup tables and gives an acceptable operating frequency. In contrast, the carry-select adder has the highest frequency at the cost of little increase in lookup tables. Therefore, for the application needing more frequency, the carryselect adder is the best choice.

\subsection{FPGA Based Implementations of Various Multiplier Circuits in 3-bit and 8-bit Configuration}

Multiplication is an important fundamental function in arithmetic operations. For multiplication algorithms performed in DSP applications, latency and throughput are the two major concerns for efficient implementation. Area-speed and power are the trade-offs for selecting the multiplier schemes among all existing architectures. Besides, the operands' input length also has an effect on the overall performance of the multiplier.

In this work, multipliers are implemented in two configurations: 3-bit and 8-bit. As the FPGA works

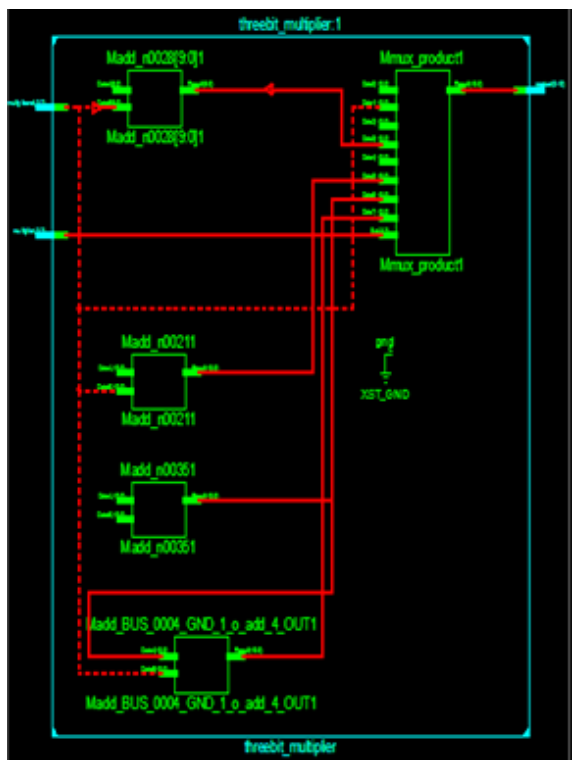

Fig. 9: RTL view of 3-bit memory based multiplier

\begin{tabular}{|l|l|l|l|}
\hline Factor & $\begin{array}{l}\text { BRAM } \\
\text { based } \\
\text { design }\end{array}$ & DSP48 & $\begin{array}{l}\text { Proposed LUT } \\
\text { based design }\end{array}$ \\
\hline LUTs & 3 & 0 & 9 \\
\hline DSP48 & 0 & 1 & 0 \\
\hline Block Ram & 9 & 0 & 0 \\
\hline Mux & 0 & 0 & 85 \\
\hline Delay (ns) & 4.372 & 9.173 & 3.648 \\
\hline Logic Level & 1 & 1 & 3 \\
\hline Frequency (MHz) & 228.7 & 109 & 274.1 \\
\hline
\end{tabular}

TABLE 3: FPGA based implementation results of built-in primitive and LUT based multiplier [15]

in a modular approach, it causes ease to implement a large circuit by instantiating the small modules. The proposed 3-bit multiplier is used for the design of an 8-bit multiplier that may further be extended to $N$-bits. The RTL view of the 3 -bit memory-based multiplier is shown in Figure 9, and the FPGA-based implementation results are given in Table 3. In Table 3 , three designs are shown and compared that include the block-RAM based architecture, FPGA's built-in primitive based design, and the proposed lookup table based design. When compared with the BRM (a chunk of memory mounted on the FPGA board for on-chip storage of a large data), and DSP48 (an ALU mounted on FPFA's fabric for performing addition, subtraction, and multiplication) based design, it may be observed that the proposed lookup table based design gives a good operating frequency with a small increase in consumed lookup tables. Hence, when dealing with the small coefficient multiplier, such as 3-bit, it is a good choice to select the proposed lookup table based design. Another 3-bit proposed multiplier is 


\begin{tabular}{|l|l|l|}
\hline Factor & $\begin{array}{l}\text { Conventional } \\
\text { shift and add }\end{array}$ & $\begin{array}{l}\text { Proposed shift } \\
\text { and add }\end{array}$ \\
\hline LUTs & 11 & 6 \\
\hline Delay $(\mathrm{ns})$ & 9.049 & 5.684 \\
\hline Logic & 7 & 3 \\
Level & & 175 \\
\hline Frequency $(\mathrm{MHz})$ & 110 & \multicolumn{2}{|l}{} \\
\hline
\end{tabular}

TABLE 4: FPGA based implementation results of conventional and proposed 3-bit shift and add multiplier algorithm [16]

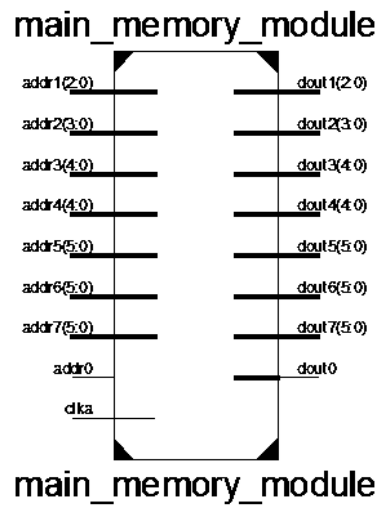

Fig. 10: RTL view of 8-bit Memory Based Multiplier

the shift and add. It is one of the easiest methods of multiplication, but when implemented on FPGA, it consumes more resources. The optimization in the conventional method may reduce the resources to some extent, which is evident from Table 4. From the results, it may be observed that the proposed shift and addbased design outperforms in achieved frequency along with a reduced number of lookup tables. Along with 3bit multipliers, 8-bit multipliers are also compared and analyzed in terms of various performance parameters. RTL view of the proposed memory-based multiplier is shown in Figure 10. The FPGA-based implementation result is shown in Table 5. The parameters compared include the achieved operating frequency and the consumed area in terms of lookup tables and other primitives. The pipeline approach uses intermediate registers for data transfer which causes the achieved frequency to be very high that is evident from the results. The Booth consumes fewer lookup tables due to less number of operations performed as compared to other approaches; hence, when the requirement is of signed multiplier and the compact design in terms of area, the Booth may be a good choice. On the other hand, the two proposed multipliers in an 8-bit configuration are comparable to other conventional approaches.

\subsection{FPGA Based Implementations of FIR Filter Using Conventional and Proposed Multipliers}

Table 6 shows the implementation of the FIR filter using the traditional and proposed multipliers. Like in standalone implementation, the pipelined multiplier achieves superior performance in terms of achieved frequency, and the Boot consumes fewer lookup tables. Whereas, the operating frequency of the proposed shift and add is also much better than the conventional shift and add multiplier. Hence the area-speed tradeoff amongst the pipeline approach and Booth is evident from the results.

\subsection{FPGA Based Implementation of LMS Filter Using the Conventional and Proposed Multiplier}

For the application of an adaptive channel equalizer, a single-coefficient LMS filter is designed and analyzed using the state-of-the-art multipliers along with FPGA's built-in DSP-48 core. The results are reported in Table 7 . It is evident from the results that the pipelined multiplier performes better in speed (achieved frequency). Nevertheless, DSP48 on the other hand consumes a minimal amount of lookup tables.

\section{Power Analysis Using Xilinx Power Ana- lyzer Tool}

Power requirements for programmable logic devices are highly dependent on the logic and functionality of the design. Early in the design cycle, we can enter the estimated activity and resources needed for our design in the Xilinx Power Estimator (XPE), which allows us to ensure that our design meets power requirements. Tables 8-9 show the power consumed in various elements of the circuits.

\section{Conclusion \& Future Work}

For the design and implementation of complex DSP systems, it is observed that multiplier is the most complex part. Various optimized architectures of multipliers are seen so far. The research shows various implementations and comparative analyses of that multiplier algorithm on FPGA, but the area-speed tradeoff is also observed. This work aims to compare the existing multipliers with the two proposed algorithms and perform their area-performance analysis. Besides, the theme of this work is also to propose the most suitable multiplier used in the design of complex DSP systems, such as adaptive channel equalizers for the application of mobile communications. 


\begin{tabular}{|l|l|l|l|l|l|l|l|l|}
\hline Factors & Shift \& Add & Serial & Booth & Wallace & Array & Pipeline & *PSA & *PLB \\
\hline LUTs & 86 & 189 & 35 & 110 & 112 & 55 & 73 & 143 \\
\hline Delay & 5.54 & 11.129 & 2.825 & 19.959 & 24.852 & 1.415 & 13.490 & 15.368 \\
& 3 & & & & & & & \\
\hline Logic Level & 5 & 32 & 10 & 15 & 23 & 0 & 13 & 16 \\
\hline Frequency & 353 & 89.8 & 353 & 50.005 & 40.022 & 505.5 & 74.6 & 65.07 \\
\hline $\begin{array}{l}\text { *PSA: Proposed Shift and Add } \\
\text { *PLB: Proposed LUT based }\end{array}$
\end{tabular}

TABLE 5: FPGA based implementation results of 8 bit conventional and proposed multipliers

\begin{tabular}{|l|l|l|l|l|l|l|l|l|}
\hline Factors & Shift \& Add & Serial & Booth & Wallace & Array & Pipeline & *PSA & *PLB \\
\hline LUTs & 5035 & 5895 & 1552 & 4305 & 3800 & 5418 & 3074 & 5472 \\
\hline Delay & 15.4 & 11.228 & 3.015 & 22.594 & 28.350 & 1.415 & 9.078 & 20.651 \\
\hline Logic Level & 29 & 32 & 10 & 19 & 25 & 0 & 27 & 22 \\
\hline Frequency & 54.5 & 89.05 & 331.50 & 43.8 & 35 & 505.51 & 110.1 & 48.8 \\
\hline
\end{tabular}

TABLE 6: FPGA based implementation results of 11 tap FIR filter using conventional and proposed multipliers

\begin{tabular}{|l|l|l|l|l|l|l|l|l|}
\hline Factors & DSP & Serial & Booth & Wallace & Array & Pipeline & ${ }^{*}$ PSA & ${ }^{* P L B}$ \\
\hline LUTs & 42 & 505 & 225 & 4305 & 3800 & 340 & 391 & 558 \\
\hline Delay & $\begin{array}{l}10.0 \\
05\end{array}$ & 5.042 & 2.455 & 22.594 & 28.350 & 1.415 & 18.065 & 18.226 \\
\hline Logic Level & 21 & 21 & 10 & 19 & 25 & 0 & 25 & 28 \\
\hline Frequency & 99.9 & 59.40 & 404.04 & 43.8 & 35 & 505.5 & 55.354 & 54.868 \\
\hline
\end{tabular}

TABLE 7: FPGA based implementation results of single coefficient LMS filter using conventional and proposed multipliers

\begin{tabular}{|l|l|l|l|l|l|}
\hline \multicolumn{1}{|c|}{$\begin{array}{c}\text { Design } \\
\text { Element }\end{array}$} & \multicolumn{5}{|c|}{ On-chip power (watts) } \\
\cline { 2 - 6 } $\begin{array}{l}\text { Carry skip } \\
\text { adder }\end{array}$ & Logic & Signal & IOs & leakage & Total \\
\hline $\begin{array}{l}\text { Carry select } \\
\text { adder }\end{array}$ & 0.002 & 0.002 & 0.004 & 0.011 & 0.019 \\
\hline $\begin{array}{l}\text { Carry look } \\
\text { ahead adder }\end{array}$ & 0.001 & 0.001 & 0.001 & 0.013 & 0.014 \\
\hline $\begin{array}{l}\text { Carry save } \\
\text { adder }\end{array}$ & 0.002 & 0.001 & 0.002 & 0.012 & 0.017 \\
\hline
\end{tabular}

TABLE 8: power analysis of various adders

\begin{tabular}{|l|l|l|l|l|l|}
\hline \multicolumn{1}{|c|}{$\begin{array}{c}\text { Design } \\
\text { Element }\end{array}$} & \multicolumn{5}{|c|}{ On-chip power (watts) } \\
\cline { 2 - 6 } & Logic & Signal & IOs & leakage & Total \\
\hline $\begin{array}{l}\text { Shift \& } \\
\text { Add }\end{array}$ & 0.001 & 0.0001 & 0.002 & 0.020 & 0.024 \\
\hline Serial & 0.000 & 0.001 & 0.000 & 0.020 & 0.021 \\
\hline Booth & 0.001 & 0.001 & 0.002 & 0.021 & 0.025 \\
\hline Wallace & 0.002 & 0.001 & 0.002 & 0.014 & 0.019 \\
\hline Array & 0.002 & 0.002 & 0.002 & 0.019 & 0.025 \\
\hline Pipeline & 0.002 & 0.002 & 0.001 & 0.018 & 0.023 \\
\hline $\begin{array}{l}\text { Proposed } \\
\text { shift and } \\
\text { add }\end{array}$ & 0.002 & 0.002 & 0.002 & 0.015 & 0.021 \\
\hline $\begin{array}{l}\text { Proposed } \\
\text { LUT based } \\
\text { design }\end{array}$ & 0.002 & 0.002 & 0.001 & 0.015 & 0.020 \\
\hline
\end{tabular}

TABLE 9: Power analysis of various multipliers
The analysis show that amongst the adder circuits, the carry select adder is better in terms of maximum achieved frequency; while the one that consumes less area is the carry look-ahead adder. When we see 3-bit multipliers, look-up table based multiplier (proposed by the authors) is good in speed when compared with conventional once; while the proposed shift and add consumes less number of resources than others. For multi-bit analysis, either in stand-alone implementation or in FIR filter and adaptive channel equalizer, the pipelined multiplier performs better in terms of achieved frequency. On the other hand, the Booth multiplier consumes fewer resources than all others. Hence, this may be concluded that for a multi-bit approach, the choice may be as per the trade-off between area and speed.

In future, the proposed multipliers will be utilized in real-time applications of an adaptive channel equalizer in the domain of communication systems.

\section{Acknowledgement}

The authors wish to thank Mehran University of Engineering and Technology and Quaid-e-Awam University of Engineering Science and Technology for facilitating in this research. 


\section{References}

[1] S. R. Faraji, P. Abillama, and K. Bazargan, "Low-Cost Approximate Constant Coefficient Hybrid Binary-Unary Multiplier for DSP Applications," in 2020 IEEE 28th Annual International Symposium on Field-Programmable Custom Computing Machines (FCCM), 2020, pp. 93-101.

[2] X. Chen, G. Liu, N. Xiong, Y. Su, and G. Chen, "A survey of swarm intelligence techniques in VLSI routing problems," IEEE Access, vol. 8, pp. 26266-26292, 2020.

[3] D. Wang, T. Wu, F. Guo, Z. Hao, and H. Li, "Block Fast FIR Algorithm and Circuit Design," in Journal of Physics: Conference Series, 2021, p. 012004.

[4] A. D. Booth, "A signed binary multiplication technique," The Quarterly Journal of Mechanics and Applied Mathematics, vol. 4, pp. 236-240, 1951.

[5] C. S. Wallace, "A suggestion for a fast multiplier," IEEE Transactions on electronic Computers, pp. 14-17, 1964.

[6] L. Dadda, "Some schemes for parallel multipliers," Alta frequenza, vol. 34, pp. 349-356, 1965.

[7] J. Swami and S. BharatiKrisna, "tirthaji maharaja, vedic Mathematics or Sixteen Simple Mathematics formulae from the veda," ed: Delhi, 1965.

[8] J. Earle, "Latched carry-save adder," IBM Technical Disclosure Bulletin, vol. 7, pp. 909-910, 1965.

[9] S. Divakara, S. Patilkulkarni, and C. P. Raj, "Novel dwt/idwt architecture for $3 \mathrm{~d}$ with nine stage $2 \mathrm{~d}$ parallel processing using split distributed arithmetic," International Journal of Image and Graphics, vol. 20, p. 2050017, 2020.

[10] A. Pathan, R. Balal, T. D. Memon, and S. A. Memon, "Analysis of booth multiplier based conventional and short word length FIR filter," Mehran University Research Journal of Engineering \& Technology, vol. 37, pp. 595-602, 2018.

[11] A. Pathan, T. D. Memon, S. Keerio, and I. H. Kalwar, "FPGA Based performance analysis of multiplier policies for FIR filter," in 2016 International Conference on Advances in Electrical, Electronic and Systems Engineering (ICAEES), 2016, pp. 17-20.

[12] A. Pathan and T. D. Memon, "A Correlation-Less Approach Towards Adaptive Channel Equalizer Based on Wiener-Hopf Equation," Wireless Personal Communications, pp. 1-10, 2021.

[13] W. Li, E. Guillena, G. Montoro, and P. L. Gilabert, "FPGA Implementation of Memory-Based Digital Predistorters with High-Level Synthesis," in 2021 IEEE Topical Conference on RF/Microwave Power Amplifiers for Radio and Wireless Applications (PAWR), 2021, pp. 37-40.

[14] G. Abbot and D. Sharma, "Modified Efficient OMS LUTDesign for Memory-Based Multiplication," Available at SSRN 3562979, 2020.

[15] J.-I. Guo, C.-M. Liu, and C.-W. Jen, "The efficient memory-based VLSI array designs for DFT and DCT," IEEE Transactions on Circuits and Systems II: Analog and Digital Signal Processing, vol. 39, pp. 723-733, 1992.

[16] D.-F. Chiper, "A systolic array algorithm for an efficient unified memory-based implementation of the inverse discrete cosine and sine transforms," in Proceedings 1999 International Conference on Image Processing (Cat. 99CH36348), 1999, pp. 764-768.

[17] D.-F. Chiper, M. S. Swamy, M. O. Ahmad, and T. Stouraitis, "Systolic algorithms and a memory-based design approach for a unified architecture for the computation of DCT/DST/IDCT/IDST," IEEE Transactions on Circuits and Systems I: Regular Papers, vol. 52, pp. 1125-1137, 2005.

[18] P. K. Meher and M. Swamy, "New systolic algorithm and array architecture for prime-length discrete sine transform,"
IEEE Transactions on Circuits and Systems II: Express Briefs, vol. 54, pp. 262-266, 2007.

[19] P. K. Meher, J. C. Patra, and M. Swamy, "Highthroughput memory-based architecture for DHT using a new convolutional formulation," IEEE Transactions on Circuits and Systems II: Express Briefs, vol. 54, pp. 606-610, 2007.

[20] P. K. Meher, "Low-latency hardware-efficient memorybased design for large-order FIR digital filters," in 2007 6th International Conference on Information, Communications \& Signal Processing, 2007, pp. 1-4.

[21] P. K. Meher, "New approach to LUT implementation and accumulation for memory-based multiplication," in 2009 IEEE International Symposium on Circuits and Systems, 2009, pp. 453-456.

[22] S. A. White, "Applications of distributed arithmetic to digital signal processing: A tutorial review," IEEE Assp Magazine, vol. 6, pp. 4-19, 1989.

[23] Y.-H. Chan and W.-C. Siu, "On the realization of discrete cosine transform using the distributed arithmetic," IEEE Transactions on Circuits and Systems I: Fundamental Theory and Applications, vol. 39, pp. 705-712, 1992.

[24] H.-C. Chen, J.-I. Guo, T.-S. Chang, and C.-W. Jen, "A memory-efficient realization of cyclic convolution and its application to discrete cosine transform," IEEE transactions on Circuits and Systems for Video Technology, vol. 15, pp. 445-453, 2005.

[25] P. K. Meher, "Unified systolic-like architecture for DCT and DST using distributed arithmetic," IEEE Transactions on Circuits and Systems I: Regular Papers, vol. 53, pp. 26562663, 2006.

[26] J.-P. Choi, S.-C. Shin, and J.-G. Chung, "Efficient ROM size reduction for distributed arithmetic," in 2000 IEEE International Symposium on Circuits and Systems. Emerging Technologies for the 21st Century. Proceedings (IEEE Cat No. 00CH36353), 2000, pp. 61-64.

[27] H.-R. Lee, C.-W. Jen, and C.-M. Liu, "On the design automation of the memory-based VLSI architectures for FIR filters," IEEE transactions on consumer electronics, vol. 39, pp. 619-629, 1993.

[28] B. K. Mohanty and P. K. Meher, "An Efficient Parallel DABased Fixed-Width Design for Approximate Inner-Product Computation," IEEE Transactions on Very Large Scale Integration (VLSI) Systems, vol. 28, pp. 1221-1229, 2020.

[29] K. Chapman, "Fast integer multipliers fit in FPGAs," EDN magazinee's Desighn Ideas, www. ednmag. com, 1993.

[30] T. D. Memon and A. Pathan, "An approach to LUT based multiplier for short word length DSP systems," in $2018 \mathrm{In}-$ ternational Conference on Signals and Systems (ICSigSys), 2018, pp. 276-280.

[31] Y. Voronenko and M. Püschel, "Multiplierless multiple constant multiplication," ACM Transactions on Algorithms (TALG), vol. 3, p. 11, 2007.

[32] S. Mirzaei, A. Hosangadi, and R. Kastner, "FPGA implementation of high speed FIR filters using add and shift method," in 2006 International Conference on Computer Design, 2006, pp. 308-313.

[33] B. Parhami, Computer arithmetic vol. 20: Oxford university press, 2010.

[34] M. Aly and A. Sayed, "A study of signed multipliers on FPGAs," in 2012 IEEE International Conference on Electronics Design, Systems and Applications (ICEDSA), 2012, pp. 33-38.

[35] A. Pathan and T. D. Memon, "An optimised $3 \times 3$ shift and add multiplier on FPGA," in 2017 14th International 
Bhurban Conference on Applied Sciences and Technology (IBCAST), 2017, pp. 346-350.

[36] T. P. Zieliński, "FIR Filters in Signal Interpolation, Resampling, and Multi-Rate Processing," in Starting Digital Signal Processing in Telecommunication Engineering, ed: Springer, 2021, pp. 257-292. 